\title{
Active Resistance to Apple Scab
}

\author{
By L. D. HUNTER, D. S. KIRKHAM AND R. C. HIGNETT \\ East Malling Research Station, Maidstone, Kent
}

(Accepted for publication 12 March 1968 )

\begin{abstract}
SUMMARY
Two partially characterized groups of compounds were isolated from an apple variety (Miller's Seedling) which has polygenic resistance to apple scab. These compounds inhibited germination of conidia of Venturia inaequalis and interfered with pigment production in log-phase cultures of the organism. They were more abundant in varieties of apple resistant to scab than in susceptible varieties, and increased in the leaves of resistant varieties after inoculation with $V$. inaequalis conidia. When applied to the foliage of a variety of apple normally susceptible to scab these compounds conferred a measure of protection against the disease. The subject of physiological resistance of plants to microbial attack is discussed.
\end{abstract}

\section{INTRODUCTION}

Previous work at East Malling has shown the importance of the part played in the plant/disease syndrome Malus sp./Venturia inaequalis by certain of the simple appleleaf phenolic substances and their analogues (Kirkham, 1957; Kirkham \& Hunter, 1965). More recently the specific effects on the transport of solutes in the host by extracellular melanoproteins produced by the pathogen has been studied (Hignett \& Kirkham, 1967). The present paper discusses some further host factors which have been found in a resistant apple variety. Comparison of extracts of apple leaves by chromatography or high voltage electrophoresis showed that varieties having a high degree of field resistance to the apple scab organism contained a number of compounds not observed in varieties which are susceptible to scab. The most notable compounds were present in two bands that fluoresced bright light-blue, in the presence of ammonia vapour, under ultraviolet radiation $(3650 \AA)$. These, for convenience, have been called Blue I and Blue II. Methods have been evolved for their isolation from apple leaves, particularly those of the variety Miller's Seedling.

\section{METHODS}

Experiments were made with plants of Miller's Seedling worked on MM. 104 rootstocks, or with rooted shoots of the MM. Iog clone. The host material and fungus were handled and trials recorded as before (Kirkham \& Hunter, 1965). Pigment production in deep culture was followed by the methods already reported (Hignett \& Kirkham, 1967). Young leaves were obtained from plants grown either in greenhouses or in orchards.

An extract of 'phenolics' was made by macerating $100 \mathrm{~g}$. leaves in a blender with $200 \mathrm{ml}$. ice-cold disodium hydrogen phosphate $(0.2 \mathrm{M}, \mathrm{pH} 8)$ containing cysteine 
hydrochloride $(0.005 \mathrm{M})$ to reduce polyphenoloxidase activity (Walker, 1964). The extract was filtered through two thicknesses of fine muslin and centrifuged at $7000 \mathrm{~g}$ and $5^{\circ}$ for $15 \mathrm{~min}$. The clear, pale yellow-green supernatant fluid was decanted, the precipitate being discarded. The supernatant fluid was extracted several times with ethyl acetate; the ethyl acetate layer (containing chlorophylls, phloridzin, phloretin, chlorogenic acid) was discarded, and the aqueous residue concentrated to a syrup on a rotary evaporator under water-pump vacuum at $35^{\circ}$. The syrup was dialysed against distilled water for $12-18 \mathrm{hr}$ in a cold room at $5^{\circ}$. The diffusate, which consisted mainly of compounds Blue I and Blue II, together with the lower molecular weight sugar, phenolic, amino acid and peptide components of apple, was evaporated to small volume.

In an alternative method leaves were macerated in $50 \%(\mathrm{v} / \mathrm{v})$ aqueous methanol. After filtration the extract was acidified $(\mathrm{pH} 5)$ with acetic acid and concentrated under vacuum on a rotary evaporator. The water-soluble components were decanted from the precipitated chlorophylls and, after extraction with ethyl acetate, were worked up in the same way as the buffer extracts.

Apple phenolics including Blue I and Blue II could also be obtained from young shoots of Miller's Seedling. The dormant shoots were powdered in a hammer mill and the powder extracted with $50 \%(\mathrm{v} / \mathrm{v})$ aqueous methanol.

Several methods were used for the separation of pure Blue I and II from the crude concentrate.

Chromatography. Whatman no. I, no. 20 and 3 MM papers were used. Solvents were B.A.W.: $n$-butanol + acetic acid + water $(63+10+27$, by volume); P.E.w. $n$-propanol + ethyl acetate + water $(7+2+\mathrm{I}$, by volume); and $2 \%$ acetic acid. Formic acid $(2 \%)$ was used to develop thin-layer chromatograms, with Schleicher \& Schull cellulose powder no. I $45(0.5 \mathrm{~mm}$.) as the adsorbent.

Electrophoresis. Phosphate buffer ( $\mathrm{pH} 7$; equal volumes $0.02 \mathrm{M}-\mathrm{NaH}_{2} \mathrm{PO}_{4}+0.02 \mathrm{M}-$ $\mathrm{Na}_{2} \mathrm{HPO}_{4}$ ) or morpholine + acetic acid buffer $(\mathrm{pH} 8 ; \mathrm{I} \cdot \mathrm{I} \%+0.6 \%$, by vol.) was used with Whatman $3 \mathrm{MM}$ paper at $26 \cdot 7 \mathrm{~V} . / \mathrm{cm}$.

Electrophoresis or chromatography gave distinct spots or bands which could be detected under ultraviolet radiation $(3650 \AA)$ in the presence of ammonia vapour. The spots or bands which fluoresced bright light-blue were cut from the paper, eluted with distilled water, the eluates concentrated, and stored at $0^{\circ}$.

Gel filtration on columns of either Sephadex G Io or Bio-Gel $P_{2}$ was of limited use for the separation. Sephadex tended to absorb materials irreversibly from the plant extract and thus decreased the efficiency and loading capacity of the column. Bio-Gel did not give a clean separation between the components of the mixture. Changing the solvent from chloroform-saturated distilled water to $2 \%$ acetic acid or weak salt solutions did not improve the separation.

\section{RESULTS}

\section{Biological activity of compounds Blue I and Blue II}

In vitro. The effects of Blue I and Blue II on the germination of conidia of Venturia inaequalis were studied by using a modification of the method of Montgomery \& Moore (I937). Solutions (O.I ml. $E_{255} 4.8$ in a $\mathrm{I} \mathrm{cm}$. cell) of Blue I and Blue II were dried on glass slides and suspensions ( $O .1 \mathrm{ml}$.) of conidia applied to the dry deposits. 
In the controls the conidial suspension was put on to an untreated slide. The slides were incubated at $18^{\circ}$ and observed at intervals.

The fungistatic effect (Table I) was not observed in the presence of $2 \%$ Oxoid malt extract (Batch II4) nor when the Blue I or Blue II were present in lower concentrations. Solutions of Blue I and Blue II were added to 5-day cultures of Venturia inaequalis growing in early $\log$ phase. After $\mathrm{I} 2$ days the mycelium was removed by centrifugation $(20,000 \mathrm{~g}$ for $10 \mathrm{~min}$.). The extracellular pigment was then fractionated by precipitation from the culture fluid with increasing concentrations of ethanol (Hignett \& Kirkham, 1967). The extinctions of the $\mathrm{N} 50$ fraction $(50 \%, \mathrm{v} / \mathrm{v}$, ethanol in water) and $\mathrm{N} 75$ fraction ( $75 \%, \mathrm{v} / \mathrm{v}$, ethanol in water) were compared with those of control cultures. The $\mathrm{N} 50$ pigment produced in the presence of Blue I and Blue II was not only present in larger amounts than in the control culture but was also more opaque to ultraviolet radiation when compared on a dry weight basis (Table 2). The extinction of the $\mathrm{N} 75$ pigment was unaltered but less was produced when Blue I or II were present.

\section{Table I. Effects of Blue I and Blue II on germination of conidia of Venturia inaequalis}

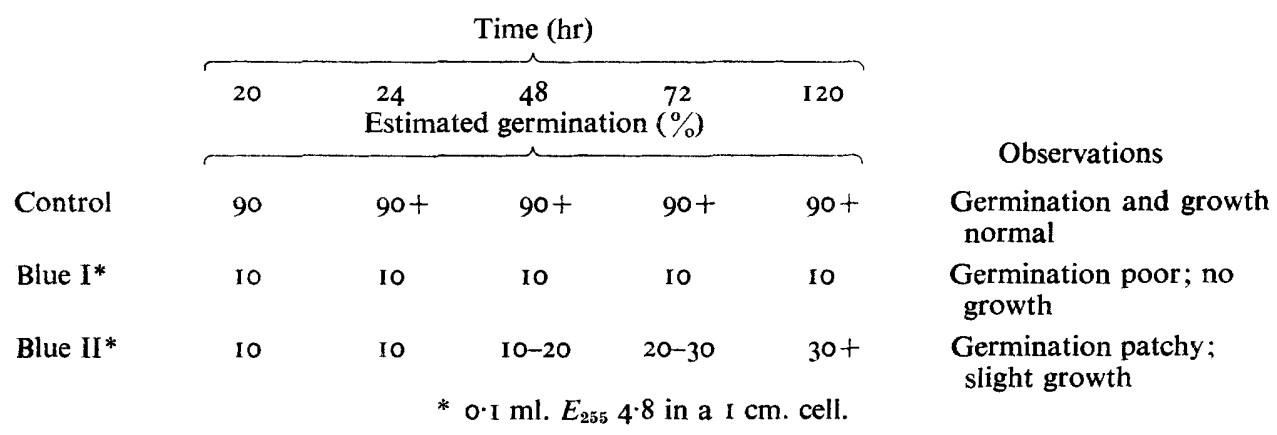

Table 2. Effects of Blue I and Blue II (Final $E_{255}$ in medium $0.72(I \mathrm{~cm}$. cell)) on pigment production by log phase cultures of Venturia inaequalis

\begin{tabular}{|c|c|c|c|c|c|c|}
\hline \multirow[b]{2}{*}{ Culture } & \multicolumn{3}{|c|}{$N_{50}$} & \multicolumn{3}{|c|}{$\mathrm{N}_{75}$} \\
\hline & $\begin{array}{l}\text { Weight } \\
\text { (mg.) }\end{array}$ & $\begin{array}{l}\text { Total } \\
\text { extinc- } \\
\text { tion* }\end{array}$ & $\begin{array}{c}\text { Total } \\
\text { extinction/ } \\
\text { mg. }\end{array}$ & $\begin{array}{l}\text { Weight } \\
\text { (mg.) }\end{array}$ & $\begin{array}{l}\text { Total } \\
\text { extinc- } \\
\text { tion* }\end{array}$ & $\begin{array}{c}\text { Total } \\
\text { extinction } \\
\text { mg. }\end{array}$ \\
\hline Control & 4.9 & $8 \cdot I$ & $\mathrm{I} \cdot 65$ & 125 & 76 & 0.605 \\
\hline Blue I added & $8 \cdot 4$ & 26 & $3 \cdot 34$ & 103 & 63 & $0.6 \mathrm{I}$ \\
\hline Blue II added & $6 \cdot 25$ & 20.4 & 3.07 & 72 & 57 & 0.79 \\
\hline
\end{tabular}

In vivo. Tests were made in a temperature-controlled greenhouse on plants of MM. I09 clonal rootstock. I. $5 \mathrm{ml}$. solutions of Blue I $\left(E_{255} 8 \cdot 0\right)$ or Blue II $\left(E_{255} 4 \cdot 8\right)$ were added to an equal volume of the inoculum which was then applied to leaves by means of an atomiser. The incidence of disease on leaves treated with inoculum to which Blue I or Blue II had been added was less than $3 \%$ lesion cover. Lesion cover on the control leaves amounted to $48 \%$.

As Blue I and Blue II had been shown to interfere with conidial germination in vitro, 
an experiment was made in which Blue I and Blue II were sprayed separately on to MM. I09 leaves at different times after the inoculum had been applied.

Figure I shows that inhibitory action of both Blue I and Blue II was restricted to a $48 \mathrm{hr}$ period after inoculation. When applied after 6 days there was no difference in degrees of infection between a Blue II treatment and the control.

Leaves of the apple cultivar Miller's Seedling were inoculated with Venturia inaequalis conidia and removed from the plants either at once or $24 \mathrm{hr}$ later. Epidermal scrapes were made on a freezing microtome stage (Kirkham \& Hunter, 1965) and

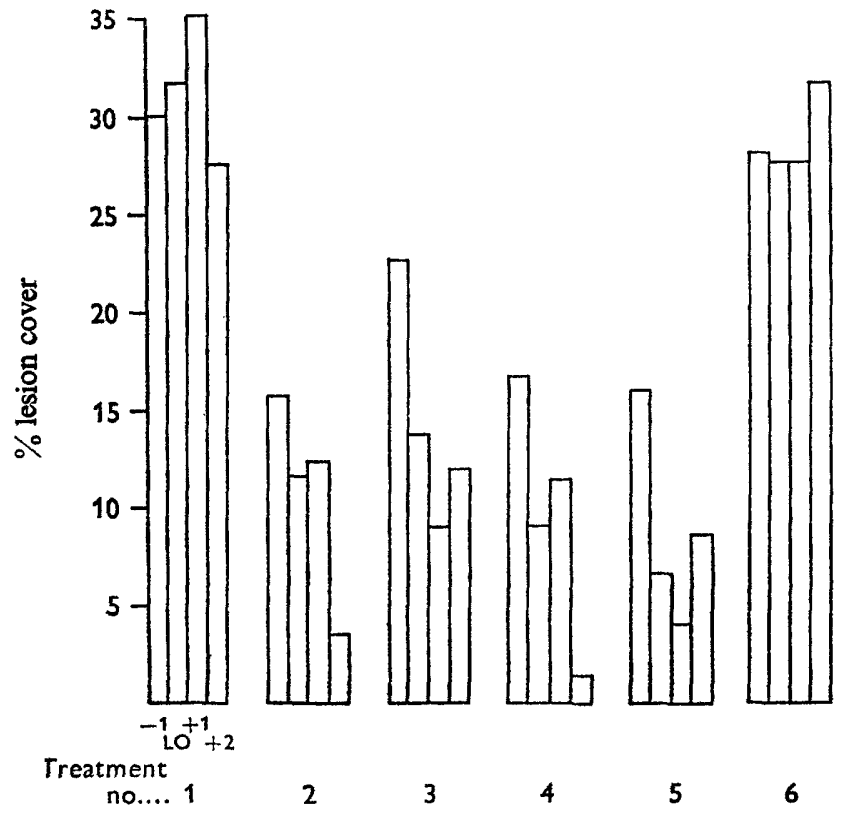

Fig. I. Effects of Blue I and Blue II [I.5 ml. $E_{255} 4.8$ (I cm. cell)] on the incidence of Venturia inaequalis on MM 109 rootstocks (four recorded leaves: $-1, L O,+I,+2$ ). (I) Control. (2) Blue I applied $24 \mathrm{hr}$ after inoculation. (3) Blue II applied $24 \mathrm{hr}$ after inoculation. (4) Blue I applied $48 \mathrm{hr}$ after inoculation. (5) Blue II applied $48 \mathrm{hr}$ after inoculation. (6) Blue II applied 6 days after inoculation.

dried in a vacuum desiccator over phosphorus pentoxide. Leaf areas sampled were in each case equivalent to $65.8 \mathrm{~cm} .{ }^{2}$ and yielded a dry sample weighing $25 \mathrm{mg}$. The dried epidermal material was shaken with $0.5 \mathrm{ml} .80 \%(\mathrm{v} / \mathrm{v})$ methanol in water, and samples of the supernatant fluid were used for thin-layer chromatography. The amounts of fluorescent components present were compared in a recording densitometer (Chromoscan) in the presence of ammonia vapour (Hignett, 1967). Although the intensity of fluorescence due to the first spot had increased twofold $24 \mathrm{hr}$ after inoculation, that due to the second spot had increased by a factor of seven and that due to the third spot by nearly four times (Table 3 ).

\section{Characterization of Blue I and Blue II compounds}

Comparison was made on thin-layer chromatograms of Blue I and Blue II, eluted after electrophoresis and used in slide tests and greenhouse trials, with the blue fluorescent material in Miller's Seedling epidermal scrapes. 
Blue I gave one rather indistinct spot, $R_{F} 0.75-0.9$, with $2 \%$ formic acid and proved to be homogeneous in a variety of other chromatographic solvents.

Blue II, on the other hand, gave three spots, $R_{F}$ values $0.8,0.7$ and 0.56 , with $2 \%$ formic acid. Rechromatography of the spot with $R_{F} 0.7$ (using the same solvent) gave two spots $R_{F}$ values 0.8 and 0.7 . Rechromatography of the spot with $R_{F} 0.56$ gave two spots $R_{F}$ values 0.8 and 0.56 . That there were two substances present, each of which existed as an equilibrium mixture of two isomers, was confirmed by electrophoresis.

Table 3. Increase in intensity of fluorescence of TLC spots obtained from Miller's Seedling leaves $24 \mathrm{hr}$ after inoculation with Venturia inaequalis

\begin{tabular}{|c|c|c|c|c|c|}
\hline & Spot & $R_{F}$ & Control* & $24 \mathrm{hr}^{*}$ & Increase $\dagger$ \\
\hline \multirow{2}{*}{ Blue I+II } & $\{1$ & 0.8 & $2 \cdot 7$ & $4 \cdot 8$ & $\times 2.4$ \\
\hline & $\{2$ & 0.7 & $I \cdot 6$ & 4.0 & $\times 7.25$ \\
\hline Blue II & 3 & 0.56 & $1 \cdot 6$ & $2 \cdot 8$ & $\times 3.75$ \\
\hline
\end{tabular}

* Area under densitometer curve in $\mathrm{cm}^{2}$

$\dagger$ Numerical results compared by method of Hignett (1967).

Comparison of the $R_{F}$ values obtained with those of the spots measured in the Miller's Seedling epidermis (Table 3 ) showed that the increase in materials contained in spot $I$ of the latter was an increase in Blue I and the faster running Blue II isomers, while increases in spots 2 and 3 were due to increased amounts of Blue II only. Thus Miller's Seedling leaves responded to invasion by Venturia inaequalis by producing large quantities of Blue I and Blue II.

Neither Blue I nor Blue II reacted with diazotized p-nitroaniline, ferric chloride, ammoniacal silver nitrate or Fehling solution. Both appeared faintly blue under ultraviolet radiation after chromatography or electrophoresis, and fluoresced bright lightblue in the presence of ammonia vapour. Determination of $\mathrm{pK}$ values was done by electrophoresis at various hydrogen ion concentrations; $\mathrm{pK}_{\mathrm{a}}$ values of 3.5 and 8.5 were found, indicating carboxyl and phenolic hydroxyl ionisations. The ultraviolet spectra of both compounds had $\lambda_{\max .} 300 \mathrm{~m} \mu$ and $\lambda_{\min .} 250 \mathrm{~m} \mu$. Sealed tube hydrolysis at $100^{\circ}$ with $5 \mathrm{~N}$-hydrochloric acid or $5 \mathrm{~N}$-sodium hydroxide gave tars and no identifiable product.

\section{DISCUSSION}

The attack of Venturia inaequalis on apple has been found to induce a complex of inter-related reactions. The pathogen, by producing melanoprotein pigments, influences the solute transport in the leaf of a susceptible variety, making the leaf a more favourable environment for growth. The response to infection which we have observed in the resistant variety Miller's Seedling is an increase in the quantities of some of the components of the phenolic fraction present in the leaves. The amounts of the substances referred to for convenience as Blue I and Blue II, for example, increased by factors from two to seven. Blue I and Blue II have been found to be detrimental to the growth of the fungus, not only in their prevention of germination of conidia, but also in their interference with normal pigment production in culture and normal growth of the fungus on leaves. This type of response to infection we propose to call 'active resistance'. This concept in the field of natural resistance of plants to microbial attack needs some clarification. Although some excellent reviews of this subject have 
been published (Walker \& Stahmann, I955; Farkas \& Kiraly, I962) some confusion has been evident of late arising mainly from the misuse of the term 'phytoalexin' and a stretching of the original concepts behind the term. In a recent paper Cruickshank (I966) quoting the hypothesis of Müller \& Börger clearly stated that: ‘ .. phytoalexin... is formed or activated only when the host cells come into contact with the parasite'. In further discussion he stated: 'Phytoalexins have not been detected in uninfected fresh plant tissues nor do they normally occur as wound responses.' This clearly defines the position and occurrence of phytoalexins. The picture is immediately clouded, however, by the section in the same paper on non-microbiological induction of phytoalexins. Under the influence of the heavy metal ions and metabolic inhibitors mentioned in that section, the production of pisatin, ipomeamarone and isocoumarin must be due to a non-specific reaction of the host. It follows, therefore, from the original definition of the term, that pisatin is not a phytoalexin, nor are ipomeamarone or isocoumarin. We believe that such a reaction to physical or chemical damage, occurring in the absence of an invading organism, should not be confused with a true host/parasite interaction and that the original definition of a phytoalexin should be strictly observed.

It may be that this effect of a parasite on the host can be closely imitated by physical or chemical means, but, considering the specificity of the host/parasite interaction and the generalized effects of the inhibitors mentioned, this appears to be unlikely. To dispel this confusion we propose the adoption of the following five classes of natural resistance of plants to attack by parasites. Here we would stress that we are considering only biochemical factors involved with fungal infections and neither morphological differences between plants nor reactions between plants and other parasites.

The first three classes are characterized by little or no biochemical interaction between host and parasite.

Passive resistance. This is shown by plants which have in their tissues a preformed fungicidal or fungistatic agent in sufficient concentration to prevent the germination of the fungus or to stop its establishment if germination and infection have taken place. The onion/Colletotrichum circinans complex is an example of this passive resistance (Walker \& Stahmann, 1955).

Wound reaction. This is the general non-specific reaction of the plant to disruptive damage of any sort which gives rise to a protective layer.

Hypersensitivity. This type of resistance is characterized by the death of the host cells at the site of invasion by the pathogen. This isolates the invading organism, which dies either from the effects of having its supply of nutrients cut off or from the action of toxins produced by the death of the host cells (Shay \& Williams, 1956, Class I reaction).

In the remaining classes of resistance there is a direct biochemical interaction between host and parasite typified by the production of antifungal compounds.

Phytoalexin production. This should be restricted to mean the production of a specialized metabolite not present in the healthy host. This metabolite (the phytoalexin) is formed by interaction between host and invading organism and is inhibitory to the latter.

Active resistance. This is the resistance of the type shown by the apple variety Miller's Seedling to Venturia inaequalis discussed in the present paper. Active resistance implies the stimulated production of fungistatic agents by the host, under the influence of the 
parasite. The agents produced differ from phytoalexins in that they are present before inoculation, and are increased in response to the pathogen. It is not yet clear whether this is a de novo synthesis or a mobilization of reserves.

The authors wish to thank H. Hutchins, A. L. Roberts, Mrs J. M. Sewell and Miss J. A. Starkey for technical assistance.

\section{REFERENCES}

Cruickshank, I. A. M. (1966). Defence mechanism in plants. Wld Rev. Pest Control 5, I6I.

FARKAS, G. L.\& KIRALY, Z. (1962). Role of phenolic compounds in the physiology of plant diseases and disease resistance. Phytopath. Z. 44, 105.

HignetT, R. C. (1967). Direct fluorimetry of phenolic compounds on thin layer chromatograms. J. Chromatog. 31, 571.

HignetT, R. C. \& KIRKHAM, D. S. (1967). The role of extracellular melanoproteins of Venturia inaequalis in host susceptibility. J. gen. Microbiol. 48, 269.

KIRKHAM, D. S. (1957). The significance of polyphenolic metabolites of apple and pear in the host relations of Venturia inaequalis and Venturia pirina. J. gen. Microbiol. 17, 49I.

KIRKham, D. S. \& HunTER, L. D. (1965). Studies of the in vivo activity of esters of $o$-coumaric and cinnamic acids against apple scab. Ann. appl. Biol. 55, 359.

MONTGOMERY, H. B.S. \& MOORE, M. H. (I937). A laboratory method for testing the toxicity of protective fungicides. J. Pomol. $\mathbf{1 5}_{5}, 253$.

Shay, J. R. \& Williams, E. B. (1956). Identification of three physiologic races of Venturia inaequalis. Phytopathology 46, 190.

Walker, J. C. \& Stahmann, M. A. (1955). Chemical nature of disease resistance in plants. A. Rev. Plant Phys. 6, 35I.

WALKER, J. R. L. (I964). Studies on the enzymic browning of apples. II. Properties of apple polyphenol-oxidase. Aust. J. Biol. Sci. 17, 360. 